\title{
Improved Postprandial Glucose with Inhaled Technosphere Insulin Compared with Insulin Aspart in Patients with Type 1 Diabetes on Multiple Daily Injections: The STAT Study
}

\author{
Halis Kaan Akturk, MD, Janet K. Snell-Bergeon, PhD, Amanda Rewers, MD, Leslie J. Klaff, MD, \\ Bruce W. Bode, MD, Anne L. Peters, MD, Timothy S. Bailey, MD, and Satish K. Garg, MD ${ }^{1}$
}

\begin{abstract}
Background: The majority of therapies have generally targeted fasting glucose control, and current mealtime insulin therapies have longer time action profiles than that of endogenously secreted insulin. The primary purpose of this study was to assess both glucose time-in-range (TIR: 70-180 mg/dL) and postprandial glucose excursions (PPGE) in 1-4 h using a real-time continuous glucose monitor (CGM) with Technosphere insulin (TI) versus insulin aspart in patients with type 1 diabetes (T1DM) on multiple daily injections (MDI).

Research Design and Methods: This pilot, investigator-led, collaborative, open-label, multicenter, clinical research trial enrolled 60 patients with T1DM with $\mathrm{HbA1c}$ levels $\geq 6.5 \%$ and $\leq 10 \%$. Individuals were randomized to treatment with titrated TI $(n=26)$ or titrated insulin aspart $(n=34)$, stratified by baseline HbA1c levels $(\leq 8 \%$ or $>8 \%)$. All were required to wear a real-time CGM throughout the trial. All patients in the TI group were advised to take supplemental inhalations at 1 and $2 \mathrm{~h}$ after meals if indicated based on postprandial glucose (PPG) values. The coprimary outcomes were assessed both in the full intent-to-treat population and in those individuals randomized to TI who were compliant with supplemental doses $\geq 90 \%$ of the time $(n=15)$. The CGM data were analyzed using linear regression models.

Results: Overall, those treated with TI versus aspart achieved comparable TIR, but less time spent in hypoglycemia $(<60$ and $<50 \mathrm{mg} / \mathrm{dL}$, both $P<0.05)$. In the TI-compliant group $(n=15)$, TIR was significantly greater $(62.5 \% \pm 2.6 \%$ vs. $53.8 \% \pm 1.7 \%, P=0.009)$ and time in hyperglycemia $>180 \mathrm{mg} / \mathrm{dL}$ was lower $(34.2 \% \pm 2.7 \%$ vs. $41.0 \% \pm 1.7 \%, P=0.045)$ as compared with the aspart group. PPG was also significantly lower in the TI cohort at 60 and 90 min postmeal, and PPGE were lower in the TI-compliant group as compared with the aspart group over $1-4$-h postmeal $(P<0.05)$. In addition, there was weight gain in the aspart group compared with weight loss in the TI group $(P=0.006)$ despite higher prandial TI insulin dose.

Conclusions: We conclude that using TI appropriately at mealtimes with supplemental dosing improves prandial glucose (TIR and 1-4h) control without any increase in time in hypoglycemia or weight gain in patients with T1DM on MDI. The study results support a larger study using a treat-to-target design to confirm these findings. Clinical trial reg. no. NCT03143816, clinicaltrials.gov.
\end{abstract}

Keywords: Technosphere Insulin, Insulin aspart, Type 1 diabetes, Postprandial hyperglycemia, Time in range, Continuous glucose monitoring.

\footnotetext{
${ }^{1}$ Barbara Davis Center for Diabetes, University of Colorado Denver, Aurora, Colorado.

${ }^{2}$ Rainier Clinical Research Center, Renton, Washington.

${ }^{3}$ Atlanta Diabetes Associates, Atlanta, Georgia.

${ }^{4}$ Keck School of Medicine of the University of Southern California, Los Angeles, California.

${ }^{5}$ AMCR Institute, Escondido, California.

Parts of this study results were presented as an oral and poster presentation at the 78th Scientific Sessions of the American Diabetes Association, Orlando, Florida, June 22-26, 2018. It is also being presented at European Association for Study of Diabetes (EASD), October 1-5, 2018, Berlin.

(c) Halis Kaan Akturk, et al., 2018; Published by Mary Ann Liebert, Inc. This Open Access article is distributed under the terms of the Creative Commons Attribution Noncommercial License (http://creativecommons.org/licenses/by-nc/4.0/) which permits any noncommercial use, distribution, and reproduction in any medium, provided the original author(s) and the source are credited.
} 


\section{Introduction}

$\mathbf{T}$ HE MAJORITY OF patients with type 1 diabetes (T1DM) are not able to achieve optimal glucose control despite advances in insulin treatment options and increasing use and application of diabetes technologies. Elevated HbA1c is well established as a measure of an increased risk of micro- and macrovascular complications and mortality. ${ }^{1-3}$ The lack of availability of an ideal rapid-acting insulin analog (RAIA) may contribute in achieving optimal glucose control, as peak effects and longer duration of action still occur with these mealtime insulins (lispro, aspart, glulisine, FiAsp ${ }^{\circledR}$ ). Limited early insulin effect (postmeal), and negligible suppression of hepatic glucose production contribute to postprandial hyperglycemia, which can result in an increased risk of delayed postprandial hypoglycemia, and higher glucose variability. ${ }^{4,5}$ Ideally, use of an ultra-rapid-acting prandial insulin that closely mimics the time action profile of normal insulin secretion and human physiology ${ }^{6}$ will lower postprandial glucose excursions (PPGE) without any delayed hypoglycemia.

Technosphere Insulin (TI, Afrezza ${ }^{\circledR}$; MannKind Corporation, Westlake Village, CA) is a dry-powder formulation of recombinant human insulin adsorbed onto Technosphere microparticles for oral inhalation. ${ }^{7}$ Furmaryl diketopiperazine, a biologically inert excipient, forms the Technosphere particle matrix. ${ }^{8,9}$ TI has a faster onset of action $(\sim 12 \mathrm{~min})$ and shorter duration of action $(\sim 2 \mathrm{~h})$ compared with the currently available RAIAs (aspart, lispro, or glulisine) with a peak effect of $35-45 \mathrm{~min}$ due in great part to its rapid absorption from the lung. ${ }^{10-12}$

An initial study using TI demonstrated noninferiority in glucose control when compared with insulin aspart in patients with T1DM using MDI. ${ }^{13}$ This registration study also documented fewer hypoglycemic events with lesser weight gain in subjects with T1DM. ${ }^{13}$ Another small pilot feasibility singlearm study (using additional inhalations after meals) showed improvement of HbAlc in 6 weeks using TI. ${ }^{14}$ However, to the best of our knowledge, no randomized clinical trials have been conducted to assess the role of additional postprandial inhalations ("treat to target design") utilizing continuous glucose monitoring (CGM) on overall glucose control, including time-in-range (TIR), prandial glucose excursions, and time in hypoglycemia. We performed this 4-week pilot investigator-led, collaborative open-label multicenter randomized clinical trial, to evaluate the effect of TI versus subcutaneous RAIA insulin (aspart) on PPG control assessed by TIR and PPGE as measured by real-time CGM.

\section{Research Design and Methods}

This study was a pilot, randomized, multicenter (five centers in the United States), 4-week active treatment trial. Adults $(\geq 18$ and $\leq 70$ years) with T1DM for at least 6 months with HbA1c 6.5\%-10.0\% were enrolled between June 2017 and December 2017. Major inclusion criteria included: nonsmoking adults, body mass index (BMI) $\leq 35 \mathrm{~kg} / \mathrm{m}^{2}$, forced expiratory volume in $1 \mathrm{~s}\left(\mathrm{FEV}_{1}\right) \geq 70 \%$ predicted, on MDI, stable insulin dose $\geq 3$ months, and using insulin degludec or glargine as basal insulin. Major exclusion criteria included: pregnancy, any pulmonary disease, severe hypoglycemia that required assistance in the last 3 months, currently smoking or marijuana use, using insulin detemir or Neutral Protamine Hagedorn
(NPH) as basal insulin, and a need for $>18 \mathrm{U}$ of prandial insulin per meal (Table 1).

At the screening visit, all patients had an Electrocardiogram (EKG), physical exam, complete metabolic panel, and spirometry tests. Spirometry $\left(\mathrm{FEV}_{1}\right.$ and FVC) was performed at baseline, and at weeks 2 and 4 during the treatment period in both groups. Safety and adverse events were assessed. Subjects who had a decrease of $\geq 20 \%$ of $\mathrm{FEV}_{1}$ at any of the follow-up study visits were withdrawn (none of the study participants met these criteria).

There were a total of seven clinic/phone visits during the study period (Fig. 1). Patients had a screening visit followed by a randomization clinic visit within 1 week. Other visits included: two additional clinic visits at weeks 2 and 4 after randomization, and four phone visits at weeks 1, 3, and 5; and 1 week following the completion of the study. This study was approved by appropriate Institutional Review Boards and monitored by an independent contract research organization. All patients provided written informed consent.

Patients were randomized 1:1 to TI or insulin aspart group using a blocked design, stratified by screening HbA1c $(\leq 8 \%$ or $>8 \%$ ). All patients used Dexcom G5 ${ }^{\circledR}$ (San Diego, CA) real-time CGM during the study period. Patients randomized to aspart continued the same bolus regimen as used before randomization. If patients were using any other RAIA (other than aspart), they were switched to aspart on the same dose at the randomization visit. Patients in the aspart group were also allowed to change their premeal bolus dose and take postprandial and other correction doses as deemed clinically necessary.

Patients randomized to TI were provided with an insulin dose conversion table (Table 2). For example, up to $4 \mathrm{U}$ of injectable RAIA was converted to $4 \mathrm{U}$ of TI. Patients taking 5-8 U of RAIA were advised to uptitrate to $8 \mathrm{U}$ of TI. Patients in the TI group were also advised to take additional inhalations at 1 and $2 \mathrm{~h}$ postprandially as indicated based on PPG values (Table 2). At $1 \mathrm{~h}$ after the meal, patients were instructed to take an additional 4 U of TI if PPG 151-200 mg/ $\mathrm{dL}$ or $8 \mathrm{U}$ if PPG was $\geq 201 \mathrm{mg} / \mathrm{dL}$. If PPG was $\geq 201 \mathrm{mg} / \mathrm{dL}$ at $2 \mathrm{~h}$, patients were instructed to take 4 additional units of TI (Table 2). During the study, patients were recommended not to change their basal insulin dose. After completion of the 4-week study, patients reverted to their standard diabetes care.

The primary efficacy endpoints were TIR for glucose and PPGE in $1-4 \mathrm{~h}$ from the CGM data. The secondary efficacy endpoints included: change in $\mathrm{HbAlc}$, under the curve (AUC) for PPG, hypoglycemia (defined as time below range: $<70,<60$, and $<50 \mathrm{mg} / \mathrm{dL}$ ), hyperglycemia (defined as time above range: $>180 \mathrm{mg} / \mathrm{dL}$ ), and glucose variability indices (standard deviation [SD] and coefficient of variation).

PPGE was defined as the difference between the highest CGM glucose level 1 to $4 \mathrm{~h}$ after a meal and the premeal CGM glucose level. ${ }^{15}$ Other glucose measurements from CGM data included: PPG, mean sensor glucose (mg/dL), AUC $(\min \times \mathrm{mg} / \mathrm{dL})$, and TIR as a percent of a 24-h day. In addition to the planned intention-to-treat comparison between the aspart and TI groups, a comparison of compliant versus noncompliant patients in the TI group was included in a posthoc per-protocol analysis, as it was noted that some study participants were not compliant with the postprandial dosing protocol. Compliance with TI use was based on using TI per protocol at 1- and 2-h postmeal based on PPG values. 
Inclusion criteria

1. Signed informed consent before any study-related activities

2. Male or female $18-70$ years of age

3. Type 1 diabetes mellitus duration more than 6 months

4. Treatment with multiple daily injections for at least 3 months before screening visit; stable insulin dose for the last 1 month

5. No use of any oral antidiabetics, any other form of insulin other than mentioned in the protocol, or any other type of injections such as GLP-1 analogs, pramlintide, or insulin/GLP-1 analog combinations

6. A1c between $6.5 \%$ and $10 \%$

7. Willingness to routinely collect at least two blood glucose measurements per day to calibrate the CGM. Beyond the calibrations, patients may use CGM for necessary action without having to confirm with fingersticks self-monitoring blood glucose, as approved by the Food and Drug Administration

8. BMI $\leq 35 \mathrm{~kg} / \mathrm{m}^{2}$

9. Ability and willingness to adhere to the protocol, including clinical and phone visits and 4-week-long CGM wear

10. Using insulin glargine or insulin degludec as basal insulin

11. Able to use and understand CGM data

12. Willing to complete phone and clinic visits

13. Patients who eat three main meals in a day (breakfast, lunch, and dinner)

14. Ability to speak, read, and write English

15. Patients prandial insulin need must be $<18 \mathrm{U}$ per meal

Exclusion criteria

1. Use of any other diabetic medication other than allowed in the protocol

2. Pregnant or intention to become pregnant during the study, or not using adequate birth control methods

3. Severe unexplained hypoglycemia requiring emergency treatment in the previous 3 months

4. Use of systemic or inhaled corticosteroids

5. History of hemoglobinopathies

6. Diagnosis of anemia

7. Postrenal transplantation, currently undergoing dialysis, creatinine $>2.0 \mathrm{mg} / \mathrm{dL}$ or a calculated creatinine clearance of $<50 \mathrm{~mL} / \mathrm{min}$

8. Advanced or unstable retinopathy needing laser procedure or vitrectomy

9. History of pancreatitis

10. Extensive skin changes/diseases that inhibit wearing a sensor on normal skin

11. Known allergy to adhesives

12. Known allergy to study medication

13. Participation in another investigational study protocol within 30 days before enrollment

14. Known chronic obstructive pulmonary disease, pulmonary hypertension, asthma, pulmonary fibrosis, or any chronic pulmonary infection, or any systemic disease that primarily affects the lungs. History of any pulmonary nodule will be excluded to participate in the study

15. Active smokers

16. Marijuana users

17. Insulin pump users

18. Expected acetaminophen use during CGM

19. Using insulin detemir or NPH as basal insulin

20. Patients who use more than $18 \mathrm{U}$ per meal

21. Any other condition, as determined by the investigator, which could make the subject unsuitable for the trial, impairs the subject's suitability for the trial, or impairs the validity of the informed consent

BMI, body mass index; CGM, continuous glucose monitor; GLP-1, glucagon-like-peptide-1; NPH, neutral protamine hagedorn.

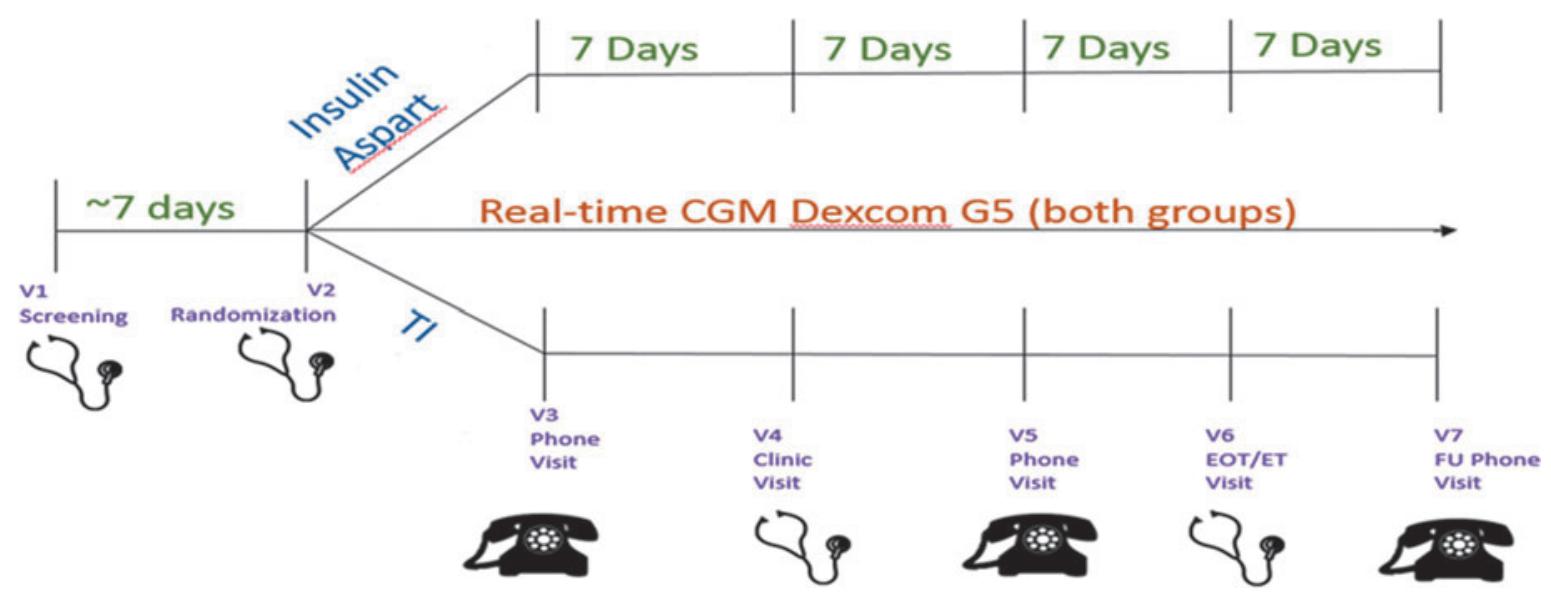

FIG. 1. Study design. 
Table 2. Injected Mealtime Insulin and Technosphere Insulin Dose Conversion Correction

\begin{tabular}{lccccc}
\hline \multicolumn{2}{c}{ TI dose conversion } & & \multicolumn{2}{c}{ TI dose correction ${ }^{\mathrm{a}}$} \\
\cline { 1 - 2 } \cline { 5 - 6 } $\begin{array}{l}\text { Injected } \\
\text { mealtime } \\
\text { insulin (units) }\end{array}$ & $\begin{array}{c}\text { Technosphere } \\
\text { Insulin dose } \\
\text { (units) }\end{array}$ & & $\begin{array}{c}\text { Blood } \\
\text { glucose } \\
\text { (mg/dL) }\end{array}$ & $\begin{array}{c}1-h \\
\text { (units) }\end{array}$ & $\begin{array}{c}2-h \\
(\text { units })\end{array}$ \\
\hline Up to 4 & 4 & & $\leq 150$ & - & - \\
$5-8$ & 8 & $151-200$ & 4 & - \\
$9-12$ & 12 & & & - \\
$13-16$ & 16 & $\geq 201$ & 8 & $4^{\mathrm{a}}$ \\
$17-20$ & 20 & & & \\
\hline
\end{tabular}

${ }^{\text {a }}$ Two-hour correction used only if blood glucose is $\geq 201 \mathrm{mg} / \mathrm{dL}$ and has not decreased by $\geq 50 \mathrm{mg} / \mathrm{dL}$ between 1 and $2 \mathrm{~h}$.

Patients were defined as compliant if $\pm 90 \%$ of postmeal TI dosages were taken per protocol, with at least one of the postmeal inhalations taken if indicated per meal. The purpose of this posthoc analysis was to generate hypotheses and plan for a future, larger clinical trial.

Baseline characteristics of the study cohort were compared by randomization group using a student's $t$-test, and CGM data were analyzed by study group using linear regression models. CGM data were aggregated per study week, to control for any changes in efficacy or compliance over time. Change in insulin bolus dose was examined in both the TI group and the aspart group; however, acknowledging that dosing between TI and aspart is not equivalent, ${ }^{16}$ these comparisons were to determine the change in bolus dosing over the course of the study.

\section{Results}

Sixty patients were randomized to TI $(n=26)$ and insulin aspart $(n=34)$. Four patients in the TI group were excluded from the final analysis (two dropped out the study due to non-side-effects-related reasons, and one had no CGM data and the other had incomplete CGM data (with the first 2 weeks of the study data missing). As shown in Table 3, baseline characteristics, including age, diabetes duration, gender, HbA1c, BMI, blood pressure, daily total basal dose, or daily total bolus dose were similar in the two groups. The baseline or end-of-the-study $\mathrm{FEV}_{1}$ did not differ by treatment

TABle 3. Baseline Characteristics

\begin{tabular}{lcc}
\hline & Aspart $(\mathrm{n}=34)$ & $T I(\mathrm{n}=26)$ \\
\hline Age (years) & $42 \pm 14$ & $41 \pm 16$ \\
Diabetes duration (years) & $19 \pm 13$ & $21 \pm 12$ \\
Screening HbA1c (\%) & $8.0 \pm 1.0$ & $7.7 \pm 0.9$ \\
Weight (kg) & $76.6 \pm 13.7$ & $80.1 \pm 11.9$ \\
Body mass index (kg/m $\left.{ }^{2}\right)$ & $25.3 \pm 2.8$ & $26.4 \pm 3.0$ \\
Systolic BP (mmHg) & $120 \pm 14$ & $127 \pm 14$ \\
Diastolic BP (mmHg) & $77 \pm 8$ & $78 \pm 8$ \\
FEV & $3.3 \pm 1.1$ & $3.4 \pm 0.9$ \\
Daily total basal dose (U/day) & $22.7 \pm 8.2$ & $27.9 \pm 15.0$ \\
Daily total bolus dose (U/day) & $21.3 \pm 8.1$ & $21.8 \pm 12.1$
\end{tabular}

Data are mean $\pm \mathrm{SD}$. Baseline characteristics were similar.

$\mathrm{BP}$, blood pressure; $\mathrm{FEV}_{1}$, forced expiratory volume in $1 \mathrm{~s} ; \mathrm{SD}$, standard deviation. group. There were no reported episodes of severe hypoglycemia in either group during the study, although four participants (all in the aspart group) reported hypoglycemia that patients were able to treat themselves. Three participants in the TI group reported mild cough, and one additional participant in the TI group reported having difficulty inhaling the TI initially, but this was resolved by drinking water (as reported by the patient).

In univariate comparisons of daily insulin dose (total basal, total bolus, premeal bolus, postprandial bolus, and correction doses) by treatment group (Table 4), the TI group reported a significantly higher total daily bolus dose when compared with the aspart group (mean \pm SD $38.5 \pm 17.2$ vs. $20.6 \pm 7.8 \mathrm{U}$ per day, respectively, $P<0.0001)$. In linear regression mixed models, daily bolus dose did not differ by study week ( $P$-value for week by treatment group interaction, $P=0.12$ ). Patients in the TI and aspart groups took a similar dose of premeal insulin $(22.7 \pm 12$ vs. $19.0 \pm 7.2 \mathrm{U}$, respectively, $P=0.17$, Fig. 2). Overall patients in the TI group took TI at 1and 2-h postprandial $27 \%$ of the time, totaling $11.4 \pm 8.2 \mathrm{U}$ of postprandial supplemental bolus doses per day. Detailed information about the insulin dosing in the TI group, including the average 1- and 2-h post-prandial (PP) dose taken per person, the percentage of time when additional doses were indicated and taken as indicated, and the average PP dose used when taken is shown in Supplementary Table S1. Meal-specific bolus doses taken by the aspart group are also shown in Supplementary Table S1. The patients in the aspart group took additional bolus doses of $1.6 \pm 1.8 \mathrm{U}$ per day. The TI group also reported taking additional doses of $4.4 \pm 5.2 \mathrm{U}$ per day, significantly higher than the additional doses reported in the aspart group (Fig. 2, $P=0.01$ ). Despite higher bolus insulin doses reported in the TI group, we observed slight but not significant weight loss in the TI group versus significant weight gain in the aspart group (weight change, TI group: $-0.22 \pm 1.3 \mathrm{~kg}$ versus aspart group: $+0.76 \pm 1.3 \mathrm{~kg}, P=0.006$ ), suggesting that the increased TI bolus insulin dose did not lead to weight gain in the TI group.

Twenty-two patients in the TI group and 34 patients in the aspart group were included in data analysis for CGM data, using linear regression models with repeated measures adjusted for age, sex, study site, study week, screening HbA1c, and total insulin dose. Glucose SD and percentage of time spent in hypoglycemia ( $<60$ and $<50 \mathrm{mg} / \mathrm{dL}$ ) were significantly lower in the TI group compared with the aspart group (Table 4). Mean sensor glucose, percentage of time in hypoglycemia $<70 \mathrm{mg} / \mathrm{dL}$, and percentage of time in hyperglycemia were all numerically lower in the TI group but the differences were not statistically significant. TIR was numerically higher but also not statistically different in the TI group (Table 4). Out of 22 patients in the TI group, 15 (68\%) were compliant and 7 were noncompliant, with average compliance in the whole group of $91 \% \pm 11 \%$. Patients in the TI-compliant group had significantly higher TIR, lower glucose SD, and less time in hyperglycemia than patients in the aspart group (Fig. 3A and Table 5, respectively). Patients in the TI-noncompliant group had significantly higher time spent in hyperglycemia ( $>180 \mathrm{mg} / \mathrm{dL}$ ) and spent less TIR when compared with compliant patients in the TI group (Table 5).

PPGE were significantly lower in the TI-compliant group than the aspart group (Fig. 3B). PPGE was significantly lower 
Table 4. Comparison of Insulin Aspart and Technosphere Insulin Groups With Intention to Treat ANALysis

\begin{tabular}{|c|c|c|c|}
\hline & Aspart $(\mathrm{n}=34)$ & $T I(\mathrm{n}=22)$ & $\mathrm{P}$ \\
\hline Final HbA1c $(\%)^{\mathrm{a}}$ & $7.8 \pm 1.0$ & $7.6 \pm 0.8$ & 0.60 \\
\hline HbA1c change $\mathrm{a}^{\mathrm{a}}$ & $-0.25 \pm 0.31$ & $-0.02 \pm 0.36$ & 0.01 \\
\hline Total basal insulin dose (units/day) ${ }^{\mathrm{a}}$ & $23.4 \pm 9.1$ & $29.0 \pm 15.3$ & 0.11 \\
\hline Total bolus insulin dose (units/day) ${ }^{\mathrm{a}}$ & $20.6 \pm 7.8$ & $38.5 \pm 17.2$ & $<0.0001$ \\
\hline Premeal dose (units/day) ${ }^{\mathrm{a}}$ & $19.0 \pm 7.2$ & $22.7 \pm 12$ & 0.17 \\
\hline Postprandial dose (units/day) ${ }^{\mathrm{a}}$ & 0 & $11.4 \pm 8.2$ & $<0.0001$ \\
\hline Correction dose (units/day) ${ }^{\mathrm{a}}$ & $1.6 \pm 1.8$ & $4.4 \pm 5.2$ & 0.02 \\
\hline Total insulin dose (units/day) ${ }^{\mathrm{a}}$ & $44.0 \pm 14.2$ & $67.7 \pm 27.2$ & $<0.0001$ \\
\hline Units per $\mathrm{kg}$ body weight/day ${ }^{\mathrm{a}}$ & $0.57 \pm 0.2$ & $0.83 \pm 0.3$ & $<0.0001$ \\
\hline Mean sensor glucose $(\mathrm{mg} / \mathrm{dL})^{\mathrm{b}}$ & $174.3 \pm 3.3$ & $168.0 \pm 4.1$ & 0.26 \\
\hline Glucose SD (mg/dL) & $66.3 \pm 1.9$ & $57.8 \pm 2.4$ & 0.01 \\
\hline$\%$ Time in range $(70-180 \mathrm{mg} / \mathrm{dL})^{\mathrm{b}}$ & $53.5 \pm 1.8$ & $58.4 \pm 2.2$ & 0.10 \\
\hline$\%$ Time in hyperglycemia $(>180 \mathrm{mg} / \mathrm{dL})^{\mathrm{b}}$ & $41.2 \pm 1.8$ & $38.1 \pm 2.3$ & 0.33 \\
\hline \%Time hypoglycemia $(<70 \mathrm{mg} / \mathrm{dL})^{\mathrm{b}}$ & $4.0 \pm 0.6$ & $2.2 \pm 0.7$ & 0.08 \\
\hline$\%$ Time in hypoglycemia $(<60 \mathrm{mg} / \mathrm{dL})^{\mathrm{b}}$ & $2.1 \pm 0.4$ & $0.7 \pm 0.5$ & 0.02 \\
\hline$\%$ Time in hypoglycemia $(<50 \mathrm{mg} / \mathrm{dL})^{\mathrm{b}}$ & $0.9 \pm 0.2$ & $0.3 \pm 0.2$ & 0.04 \\
\hline
\end{tabular}

${ }^{\mathrm{a}}$ Mean $\pm \mathrm{SD}$, unadjusted.

${ }^{\mathrm{b}} \mathrm{LS}$ mean $\pm \mathrm{SE}$, adjusted for age, sex, study site, study week, screening HbA1c, and total daily insulin dose.

in the TI-compliant group compared with the aspart group at breakfast and lunch but not at dinner (Fig. 3D).

CGM glucose SD during daytime (6 AM to midnight) was significantly lower in the TI-compliant group compared with the aspart group (Fig. 3C). However, there was no difference between groups for the CGM glucose SD during nighttime (Midnight to $6 \mathrm{AM}$ ).

The AUC for 2-h PPG was significantly lower in the TIcompliant group than the aspart group and the TInoncompliant group (Fig. 4A). As shown in Figure 4B, when

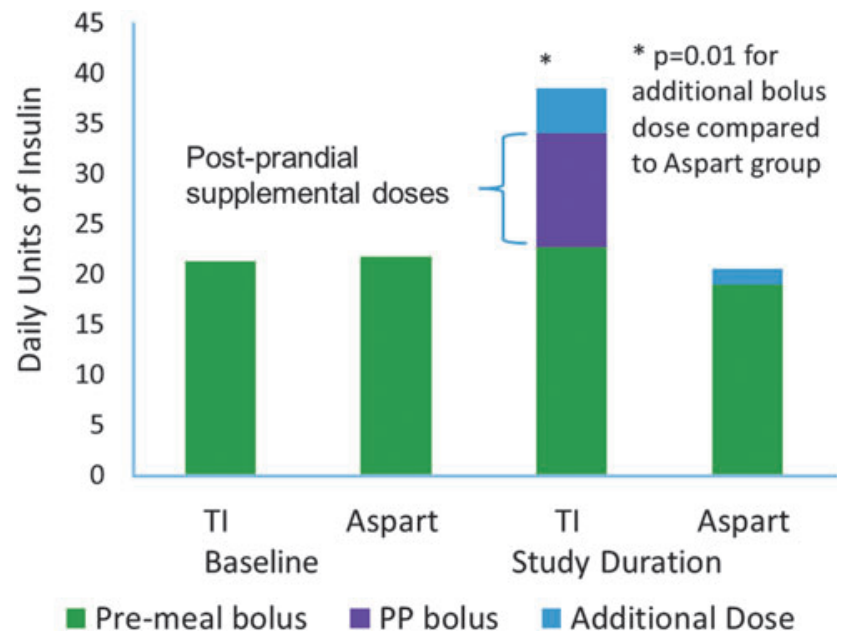

FIG. 2. Mean premeal and postprandial bolus dose, comparison of insulin aspart and TI groups. In linear regression models with repeated measures, adjusted for age, sex, study site, study week, and treatment group, the TI group used a similar premeal bolus, but a significantly larger additional bolus dose when compared with the aspart group. In addition, the TI group took planned postprandial supplemental doses per protocol (PP bolus). Average postprandial bolus was significantly higher in the TI group. PP, post-prandial; TI, Technosphere Insulin. examined by time of day, TIR was significantly higher in the TI than the aspart group during the daytime (6am-midnight) but not different at night (midnight-6am).

As shown in Figure 5, PPG values premeal did not differ between the TI group overall and the aspart group, but at 60 and 90 min after meals PPG values were significantly lower in the TI than the aspart group. At $2 \mathrm{~h}$ PPG values were also numerically lower but this trend was not statistically significant. Similarly, no significant differences at 150, 180, 210, and 240 min postmeal PPG were observed. However, when comparing the aspart group and the TI-compliant group, PPG was significantly lower in the TI-compliant group compared with the aspart group at 60,90 , and 120 min after meals (Fig. 5). There was no difference in premeal glucose level between the TI-compliant and aspart groups, but the TInoncompliant group had higher premeal PPG levels than the TI-compliant group (Fig. 5).

While there was no difference in $\mathrm{HbA} 1 \mathrm{c}$ by the study group at either screening or at the study end, participants in the aspart group had a significantly greater decrease in HbAlc than those in the TI group (Table 4) between screening and the end of the study (4 weeks). This may be due to slightly higher baseline HbAlc values in the aspart group, as the mean 24-h glucose was similar in the two groups.

\section{Discussion}

The STAT study, to the best of our knowledge, is the first to demonstrate that conversion to use of TI achieved significantly greater improvement in postprandial glucose, increased daytime TIR, decreased PPGE and hypoglycemia, and lesser overall and daytime glucose variability, although these improvements were modest. Furthermore, when used as instructed per protocol, the use of TI also increased 24-h TIR. While nighttime glucose SD were similar in both cohorts, this observation is not surprising given the short duration of action of TI.

PPGE was significantly improved in the TI group compared with the aspart group at breakfast and lunch in our 

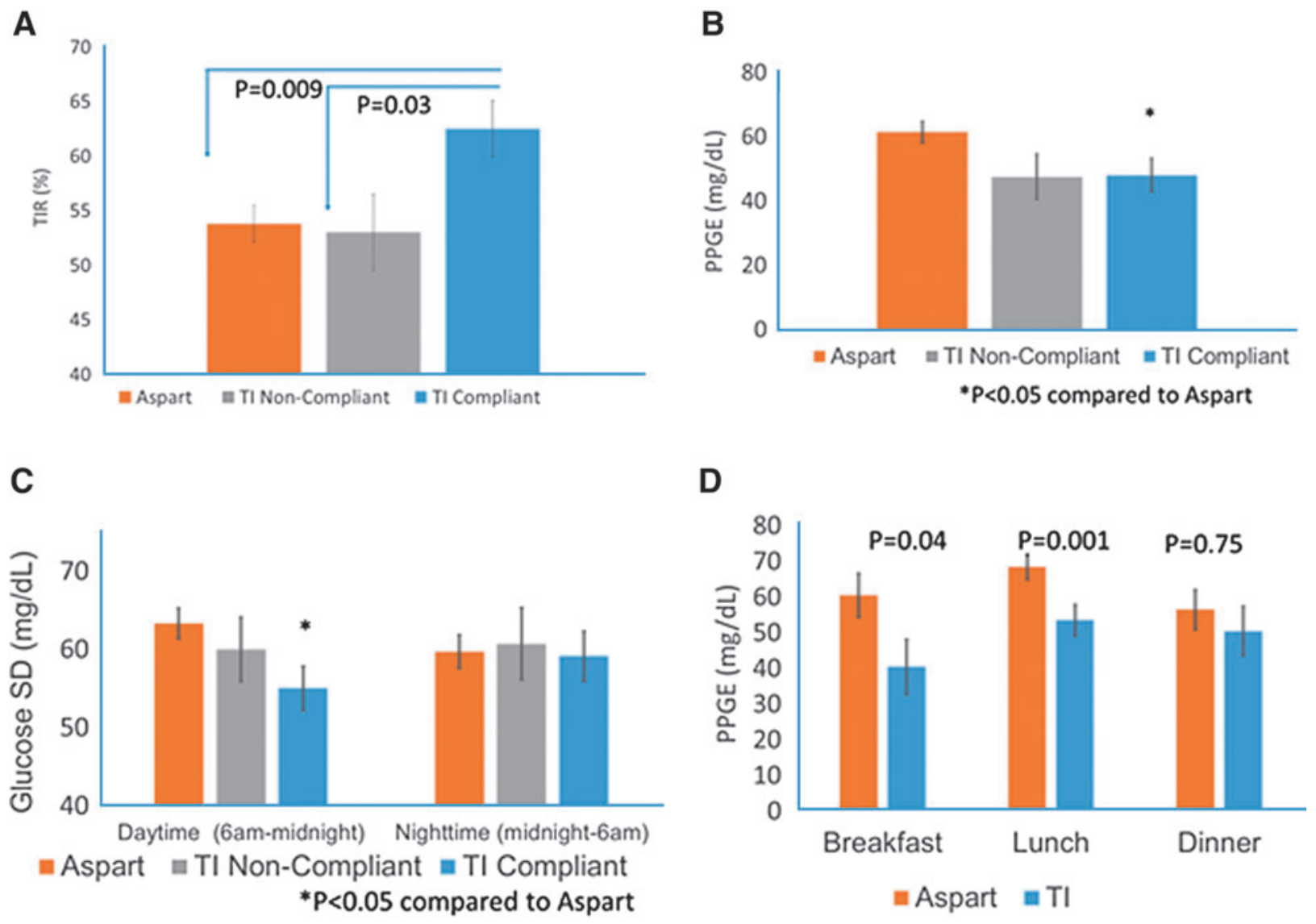

FIG. 3. Comparison of insulin aspart and TI groups for TIR, postprandial glucose excursions, and SD. (A) TIR (70$180 \mathrm{mg} / \mathrm{dL}$ ) as a percentage of a 24-h day by treatment group and compliance. TIR was significantly higher in TI-compliant group compared with TI-noncompliant and aspart groups. (B) PPGE, defined as the peak increase in CGM glucose in the 1-4 $\mathrm{h}$ after meals, in $\mathrm{mg} / \mathrm{dL}$ by treatment group and compliance. The TI-compliant group had significantly less post-prandial glucose excursion (PPGE) compared with aspart group. (C) Glucose SD during daytime (6am-midnight) and nighttime (midnight-6am) by treatment group and compliance. CGM glucose SD during daytime (6 AM to midnight) were significantly lower in the TI-compliant group compared with the aspart group. (D) PPGE, defined as the peak increase in CGM glucose in the 1-4 h after each meal, by treatment group and meal. PPGE was significantly lower in the TI-compliant group compared with the aspart group at breakfast and lunch but not at dinner. CGM, continuous glucose monitor; PPGE, post-prandial glucose excursions; SD, standard deviation; TIR, time-in-range.

Table 5. Comparison of Insulin Aspart and Technosphere Insulin Groups with Per-Protocol Analysis

\begin{tabular}{|c|c|c|c|}
\hline & Aspart $(\mathrm{n}=34)$ & TI-noncompliant $(\mathrm{n}=7)$ & TI-compliant $(\mathrm{n}=15)$ \\
\hline Total basal insulin dose (units/day) ${ }^{\mathrm{a}}$ & $23.4 \pm 2.1$ & $31.1 \pm 4.6$ & $28.2 \pm 2.9$ \\
\hline Total bolus insulin dose (units/day) ${ }^{a}$ & $20.6 \pm 2.1$ & $33.6 \pm 4.7^{b}$ & $40.5 \pm 3.0^{b}$ \\
\hline Premeal dose (units/day) ${ }^{\mathrm{a}}$ & $19.0 \pm 1.6$ & $20.6 \pm 3.5$ & $23.6 \pm 2.3$ \\
\hline Postprandial dose (units/day) ${ }^{\mathrm{a}}$ & 0 & $10.9 \pm 1.8^{b}$ & $11.5 \pm 1.2^{\mathrm{b}}$ \\
\hline Correction dose (units/day) ${ }^{\mathrm{a}}$ & $1.6 \pm 0.6$ & $2.1 \pm 1.3^{\mathrm{c}}$ & $5.4 \pm 0.9^{b}$ \\
\hline Total insulin dose (units/day) ${ }^{\mathrm{a}}$ & $44.0 \pm 3.6$ & $64.8 \pm 7.8^{\mathrm{b}}$ & $68.9 \pm 5.0^{\mathrm{b}}$ \\
\hline Units per $\mathrm{kg}$ body weight/day ${ }^{\mathrm{a}}$ & $0.57 \pm 0.04$ & $0.82 \pm 0.08^{b}$ & $0.83 \pm 0.05^{\mathrm{b}}$ \\
\hline Mean sensor glucose $(\mathrm{mg} / \mathrm{dL})^{\mathrm{d}}$ & $174.1 \pm 3.0$ & $173.6 \pm 6.4$ & $162.7 \pm 4.7$ \\
\hline Glucose SD $(\mathrm{mg} / \mathrm{dL})^{\mathrm{d}^{\mathrm{s}}}$ & $65.8 \pm 2.0$ & $59.3 \pm 4.3$ & $53.9 \pm 3.2^{\mathrm{b}}$ \\
\hline$\%$ Time in range $(70-180 \mathrm{mg} / \mathrm{dL})^{\mathrm{d}}$ & $53.8 \pm 1.7$ & $53.0 \pm 3.5^{\mathrm{c}}$ & $62.5 \pm 2.6^{b}$ \\
\hline$\%$ Time in hyperglycemia $(>180 \mathrm{mg} / \mathrm{dL})^{\mathrm{d}}$ & $41.0 \pm 1.7$ & $43.8 \pm 3.6^{\mathrm{c}}$ & $34.2 \pm 2.7^{b}$ \\
\hline$\%$ Time in hypoglycemia $(<70 \mathrm{mg} / \mathrm{dL})^{\mathrm{d}^{\prime}}$ & $3.8 \pm 0.6$ & $2.1 \pm 1.3$ & $2.2 \pm 1.0$ \\
\hline$\%$ Time in hypoglycemia $(<60 \mathrm{mg} / \mathrm{dL})^{\mathrm{d}}$ & $2.0 \pm 0.4$ & $0.7 \pm 0.8$ & $0.6 \pm 0.6$ \\
\hline$\%$ Time in hypoglycemia $(<50 \mathrm{mg} / \mathrm{dL})^{\mathrm{d}}$ & $0.8 \pm 0.2$ & $0.2 \pm 0.4$ & $0.2 \pm 0.3$ \\
\hline
\end{tabular}

${ }^{\mathrm{a}} \mathrm{LS}$ means $\pm \mathrm{SE}$, adjusted for age, sex, and study site.

${ }^{\mathrm{b}} P<0.05$ compared with Standard Treatment Group.

${ }^{\mathrm{c}} P<0.05$ TI-noncompliant compared with TI-compliant Group.

${ }^{\mathrm{d}} \mathrm{LS}$ means \pm SE, adjusted for age, sex, study site, and total insulin dose. 

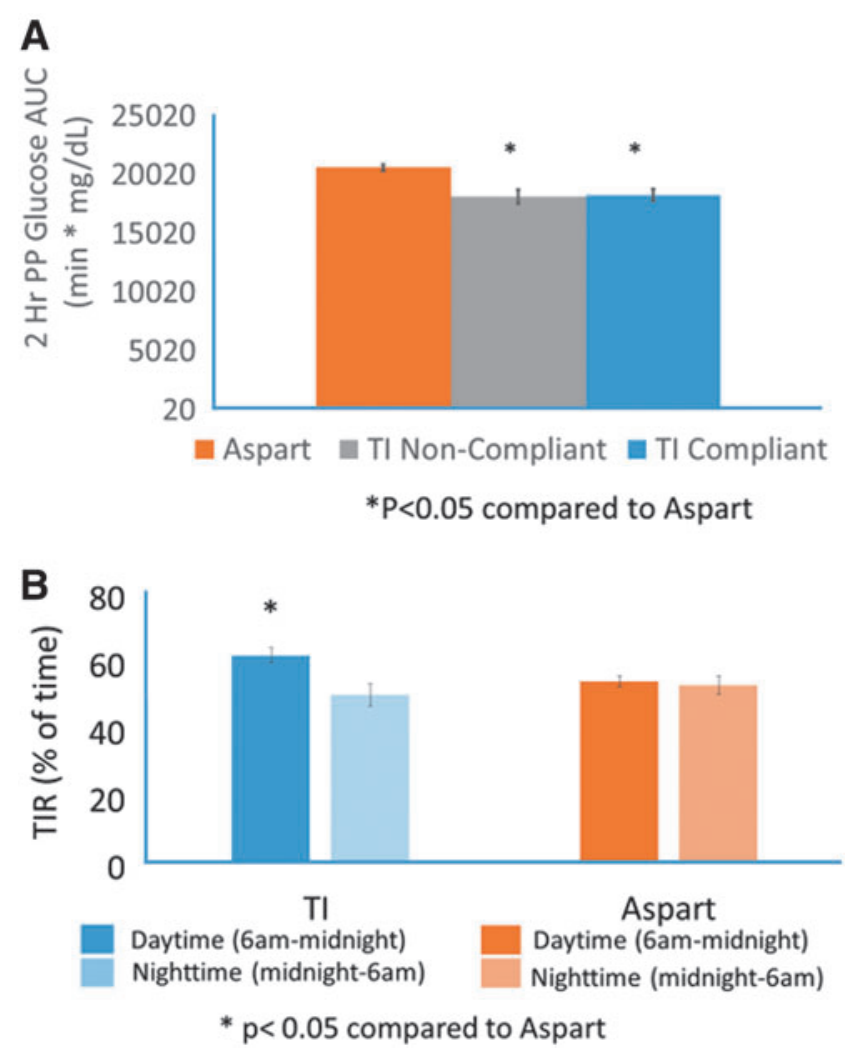

FIG. 4. 2-h Postprandial AUC and TIR by time of day, comparison of insulin aspart and TI groups. (A) The AUC for $2 \mathrm{~h}$ PPG was significantly lower in the TI-compliant and the TInoncompliant groups than the aspart group. (B) The TIR (70$180 \mathrm{mg} / \mathrm{dL}$ ) as a percentage of a $24 \mathrm{~h}$ day was significantly higher in the TI group than in the aspart group during the day, but was not different at night. AUC, area under the curve. study. However, no difference was observed at dinner in this pilot investigation. The potential reasons for this observation at dinnertime include the fear of hypoglycemia overnight-an experience that might be anticipated with an insulin with longer duration of action. However, as the vast majority of T1DM patients currently use subcutaneous RAIA, many may be reticent to use postprandial corrections later in the day. ${ }^{17,18}$ As TI has a shorter duration and a faster action profile compared with currently available mealtime injectable insulins, this may offer an advantage to both minimize the risk of nocturnal hypoglycemia and increase the use of supplemental or corrective doses before bedtime. ${ }^{11}$ Our study suggests that patients using TI may benefit from additional education and reassurance to both understand the time action profile and appropriately take advantage of the ultra-rapid action of TI after meals.

In the TI group, about a third of patients were not compliant with the study protocol for non-side-effects-related reasons. Despite significant education of the time action profile of TI as compared with aspart, patients in this study were naive to TI and this may have resulted in a reluctance to use the recommended doses. Similarly, while both aspart and TI are dosed in units, TI starting doses are currently advised to be $\sim 1.5$ times of the usual subcutaneous doses. ${ }^{16}$ The recently updated TI product label has a starting dose conversion chart and patients are recommended to titrate the dose after initiation, ${ }^{12}$ but patients naive to TI therapy may understandably be hesitant to take larger doses due to the risk of hypoglycemia and nonfamiliarity. A comparison of glucose response, measured by glucose infusion rate (GIR) showed that $12 \mathrm{U}$ of TI had a similar but faster peak compared with $8 \mathrm{U}$ of insulin lispro. ${ }^{16}$ Equivalent TI units are generally 1.5 to 2.0 times the injectable insulin units to reach a similar potency in clinical practice. ${ }^{19,20}$ Bolus doses in the TI group increased about two-

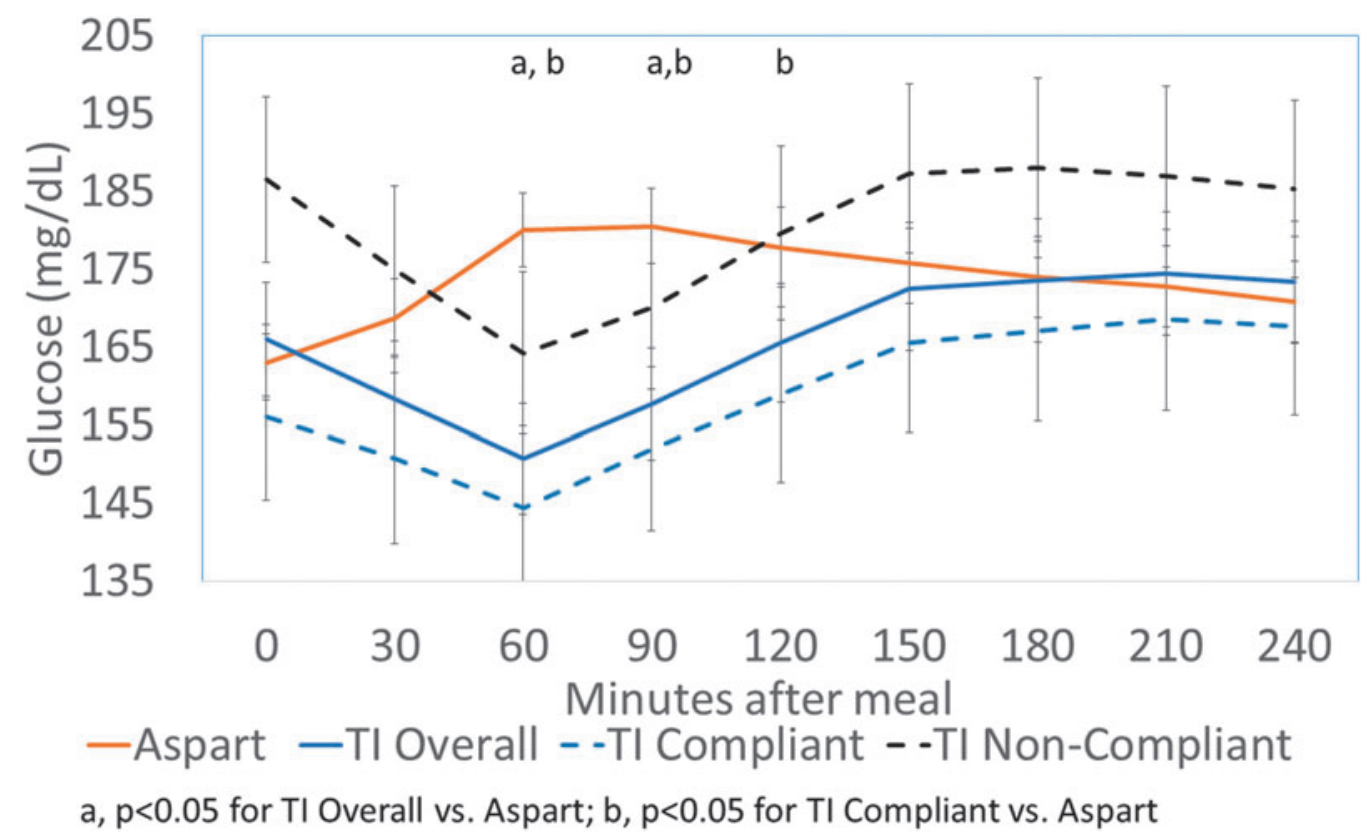

FIG. 5. Mean CGM glucose, comparison of insulin aspart and TI groups. Mean postprandial CGM glucose by treatment group and compliance. PPG values were significantly lower at 60 and $90 \mathrm{~min}$ in the TI group compared with the aspart group. PPG values were significantly lower at 60, 90, and 120 min after meals in the TI-compliant group compared with the aspart group. 
fold in this study, consistent with the expected dose conversion ratio based on GIR data.

While these data support that TI significantly improve both TIR and PPGE, the current study also has limitations. First, despite stratification of $\mathrm{HbA1c}$, patients in the aspart group had slightly, although not significantly, higher HbA1c than the TI group. This may explain the decrease in HbA1c in a short period of time (4 weeks) in the aspart group as compared with TI-treated individuals. Second, this pilot study did not have a run-in phase to optimize insulin dosing before randomization, and in addition to the study's short duration, may have limited the magnitude of differences between groups. Third, patients not used to having worn CGM in the past might have had an independent impact of CGM that is separate from the effect of TI or aspart. Fourth, this pilot study had a relatively small sample size, which may have resulted in the study being underpowered to observe some actual differences. Lastly, the high percentage of TI insulin administered postprandially suggests that higher doses of TI should have been given preprandially. While the TI algorithm used in this study led to the modest improvements (vs. aspart) described above, further studies are needed to replicate these findings and to provide guidance for more effective dosing of TI.

\section{Conclusions}

In summary, when dosed as instructed, TI significantly improved postprandial glucose without increasing hypoglycemia or weight gain as well as all day glucose TIR. Furthermore, TI decreased daytime glucose variability and reduced time spent in hypoglycemia. The faster onset of action and shorter duration profile of TI, when compared with RAIAs, may provide a flexible approach for patients to optimize postprandial glucose control without an increased risk of hypoglycemia. A larger study in insulin-requiring patients with diabetes with a treat-to-target design would provide the opportunity to confirm these findings.

\section{Acknowledgment}

This study was funded by MannKind Corporation.

\section{Author Disclosure Statement}

H.K.A. received a research grant from MannKind Corporation through the University of Colorado related to this study. S.K.G. received research grants from MannKind Corporation, Eli Lilly, Novo Nordisk, Merck, Lexicon, Medtronic, Dario, NCI, T1D Exchange, NIDDK, JDRF, Animas, Dexcom, and Sanofi through University Colorado; received consulting fees for advisory boards from MannKind, Dexcom, Eli Lilly, Novo Nordisk, Sanofi, Roche, Merck, Lexicon, and Medtronic. T.S.B. received research grant from Abbott, Ambra, Ascensia, BD, Boehringer Ingelheim, Calibra Medical, Companion Medical, Dance Biopharm, Dexcom, Eli Lilly, Glooko, Glysens, Kowa, Lexicon, MannKind, Medtronic, Novo Nordisk, Sanofi, Senseonics, Taidoc, Versartis, and Xeris; received consulting honoraria from Abbott, Astra Zeneca, Ascensia, BD, Calibra, Capillary Biomedical, Eli Lilly, Intarcia, Medtronic, Novo Nordisk, and Sanofi; and received speaking honoraria from Abbott, Eli Lilly, Medtronic, Novo Nordisk, and Sanofi. L.J.K. received research grants from Sanofi, Gan and Lee, Oramed, Lilly, Novo Nordisk, Janssen, MannKind, Dexcom, Medtronic, Senseonics, Abbott, Pfizer, and Mylan. A.P. received research support from Astra Zeneca, Dexcom, and MannKind, speaker's bureau from Novo Nordisk; participated on advisory boards for Abbott Diabetes Care, Becton Dickinson, Boehringer Ingelheim, Eli Lilly and Company, Lexicon, Livongo, Medscape, Merck, Novo Nordisk, OptumHealth, Sanofi, and Science 37. B.W.B. received research grant by Atlanta Diabetes Associates from Abbott, Becton Dickinson, Dexcom, GSK, Diasome, Jannsen, Lexicon, Lilly/Boehringer Ingelheim, MannKind, Medtronic, NIH, Novo Nordisk, Sanofi, and Senseonics; consultant fee from Adocia, Intarcia, Janssen, Medtronic, MannKind, Novo Nordisk, Sanofi; speaker's bureau from Astra Zeneca, Lily/Boehringer Ingelheim, Jannsen, Medtronic, Novo Nordisk, and Sanofi; and has stocks of Aseko. A.R. and J.S.B. do not have any conflict of interests.

\section{References}

1. Orchard TJ, Nathan DM, Zinman B, et al.: Association between 7 years of intensive treatment of type 1 diabetes and long-term mortality. JAMA 2015;313:45-53.

2. de Boer IH, Rue TC, Cleary PA, et al.: Long-term renal outcomes of patients with type 1 diabetes mellitus and microalbuminuria: an analysis of the Diabetes Control and Complications Trial/Epidemiology of Diabetes Interventions and Complications cohort. Arch Intern Med 2011; 171:412-420.

3. Nathan DM, Cleary PA, Backlund JY, et al.: Intensive diabetes treatment and cardiovascular disease in patients with type 1 diabetes. N Engl J Med 2005;353:2643-2653.

4. Ketema EB, Kibret KT: Correlation of fasting and postprandial plasma glucose with HbA1c in assessing glycemic control; systematic review and meta-analysis. Arch Public Health 2015;73:43.

5. Chen JW, Christiansen JS, Lauritzen T: Limitations to subcutaneous insulin administration in type 1 diabetes. Diabetes Obes Metab 2003;5:223-233.

6. Dailey G, Ahmad A, Polsky S, Shah V: A novel option for prandial insulin therapy: inhaled insulin. Postgrad Med 2016;128:839-847.

7. Cassidy JP, Amin N, Marino M, et al.: Insulin lung deposition and clearance following Technosphere(R) insulin inhalation powder administration. Pharm Res 2011;28: 2157-2164.

8. Pfutzner A, Mann AE, Steiner SS: Technosphere/Insulin-a new approach for effective delivery of human insulin via the pulmonary route. Diabetes Technol Ther 2002;4:589-594.

9. Potocka E, Cassidy JP, Haworth P, et al.: Pharmacokinetic characterization of the novel pulmonary delivery excipient fumaryl diketopiperazine. J Diabetes Sci Technol 2010;4: 1164-1173.

10. Heinemann L, Baughman R, Boss A, Hompesch M: Pharmacokinetic and pharmacodynamic properties of a novel inhaled insulin. J Diabetes Sci Technol 2017;11:148-156.

11. Nuffer W, Trujillo JM, Ellis SL: Technosphere insulin (Afrezza): a new, inhaled prandial insulin. Ann Pharmacother 2015;49:99-106.

12. Afrezza label information. https://www.afrezza.com/new_pi_ article/ (accessed March 16, 2018).

13. Bode BW, McGill JB, Lorber DL, et al.: Inhaled technosphere insulin compared with injected prandial insulin in type 1 diabetes: a randomized 24-week trial. Diabetes Care 2015;38:2266-2273. 
14. Garg SK, Kelly W, Freson B, Ritchie P: Treat-to-Target Technosphere[reg] Insulin in Patients with Type 1 Diabetes. American Diabetes Association, Abstract number: 941-P; 2011.

15. American Diabetes Association: Postprandial blood glucose. Diabetes Care 2001;24:775-778.

16. Heinemann L, Parkin CG: Rethinking the viability and utility of inhaled insulin in clinical practice. J Diabetes Res 2018;2018:4568903.

17. Martyn-Nemeth P, Schwarz Farabi S, Mihailescu D, et al.: Fear of hypoglycemia in adults with type 1 diabetes: impact of therapeutic advances and strategies for prevention-a review. J Diabetes Complications 2016;30: $167-177$.

18. Bohme P, Bertin E, Cosson E, Chevalier N: Fear of hypoglycaemia in patients with type 1 diabetes: do patients and diabetologists feel the same way? Diabetes Metab 2013;39:63-70.
19. Baughman RA, Heise T, Grant ML: Technosphere Insulin Inhalation Powder (TI) Displays Earlier Onset and Shorter Duration than Insulin Lispro (Lispro). American Diabetes Association 76th Scientific Sessions, New Orleans, LA, 2016.

20. Pettus J, Santos Cavaiola T, Edelman S: Recommendations for initiating use of Afrezza inhaled insulin in individuals with type 1 diabetes. Diabetes Technol Ther 2018;20:448-451.

Address correspondence to: Satish K. Garg, MD

Barbara Davis Center for Diabetes University of Colorado Denver 1775 Aurora Ct, Room 1319 Aurora, CO, 80045

E-mail: satish.garg@ucdenver.edu 